\title{
PAPER
}

\section{Thalamic proton magnetic resonance spectroscopy in vegetative state induced by traumatic brain injury}

\author{
M Uzan, S Albayram, S G R Dashti, S Aydin, M Hanci, C Kuday
}

J Neurol Neurosurg Psychiatry 2003;74:33-38

See end of article for authors' affiliations

Correspondence to: Dr M Uzan, PO Box 5, Cerrahpasa 34301, Istanbul, Turkey; uzan@istanbul.edu.tr

Received 4 September 2001 In revised form

21 August 2002

Accepted 30 August 2002

\begin{abstract}
Objectives: To determine whether proton magnetic resonance spectroscopy (MRS), a newer radiographic technology, would be useful in the evaluation of the thalamus of patients in vegetative states resulting from traumatic brain injury.

Methods: 14 victims of severe traumatic brain injury who were in the vegetative state and whose magnetic resonance images of the thalamus were normal underwent bilateral thalamic proton (MRS) studies. The $\mathrm{N}$-acetyl aspartate to creatine (NAA:Cr) and choline to creatine $(\mathrm{Cho:} \mathrm{Cr})$ ratios were obtained for each patient. The proton thalamic MRS findings of patients who were in a persistent vegetative state $(n=8)$ and in patients who had regained awareness after being in the vegetative state $(n=6)$ were compared with proton thalamic MRS findings in five healthy volunteers.

Results: While conventional magnetic resonance imaging suggested that each patient had a normal thalamus, proton MRS indicated that the thalamus of each patient in the series was damaged. The NAA: $\mathrm{Cr}$ ratio was significantly lower in the thalami of both the patients who remained in a persistent vegetative state for the duration of the study and in those who regained awareness after being in the vegetative state $(p<0.001)$. In addition, NAA: $\mathrm{Cr}$ ratios were lower in the group of patients who remained in a persistent vegetative state than in the group of patients who regained awareness after being in the vegetative state $(p<0.001)$.

Conclusions: Results suggest that the NAA:Cr ratio within the thalamus is significant and that thalamic MRS may be helpful when attempting to determine the degree of severity of neuronal and axonal injury in patients in the vegetative state.
\end{abstract}

$\mathrm{T}$ he term "vegetative state" was first used by Jennett and Plum in 1972 to describe a clinical condition of wakefulness with complete unawareness of self and environment. ${ }^{1}$ Patients in the vegetative state have sleep-wake cycles with complete or partial preservation of hypothalamic and brainstem function ${ }^{2}$ but no ascertainable cortical function. Post-traumatic vegetative state is defined as persistent if it is present for at least six months after the onset of trauma; the prognosis for these patients is poor. ${ }^{3}$ While the average life expectancy for most patients in the vegetative state ranges from 2 to 5 years, the chances of such a patient surviving longer than 15 years are extremely slim ( 1 in 15000 to 1 in $75000) .^{45}$

Several phenomena, including trauma, vascular damage, and malfunction, or hypoxic brain damage may result in the onset of the vegetative state. Some patients recover completely, while others who do regain consciousness are affected by permanent physical, cognitive, or behavioural deficits. Other patients never regain recognisable signs of awareness or the ability to respond purposefully to the environment for any significant length of time. There is neither a clinical nor an instrumental examination or medical technology that accurately predicts which patients are likely to recover from vegetative state or how much cognitive function the patient will regain. Diagnostic tests or examinations, as well as some sort of quantifiable measurement or monitoring tool, need to be developed to attempt to predict the outcome for these patients.

In patients in a persistent vegetative state and associated widespread brain damage, the association cortices, in conjunction with primary and secondary sensory cortices, is considered to be the critical neuroanatomical substrate. ${ }^{6}$ The role of the thalamus in the persistent vegetative state is defined by its highly interdependent relation to the cerebral cortex. Although the thalamus is known primarily as the receptor and storehouse of sensory information from the outside world, thalamic damage encompasses more than the destruction of this type of information. Global impairment in patients in a persistent vegetative state with disproportionately severe thalamic damage is therefore likely to be the result of damage to nuclei that are critical components of the distributed neuronal networks that underlie various cognitive and effective functions. These include attention in the external world. ${ }^{6}$ Given that the thalamus is, in a sense, a compact version of the cerebral cortex confined to a small locus and vascular distribution, it is not surprising that bilateral damage to it may result in global deficit associated with a persistent veg-

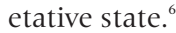

In recent years, localised proton magnetic resonance spectroscopy (MRS) has been used to monitor biochemical and metabolic changes associated with traumatic, metabolic, infectious, and tumoural pathologies of the central nervous system. ${ }^{7-11}$ In the most commonly used proton spectra, peak concentrations of various substances are known to represent specific metabolic processes. The peak for $N$-acetyl aspartate (NAA) indicates neuronal integrity, the peak for choline (Cho) represents membrane turnover, and the peak for creatine $(\mathrm{Cr})$ relates to energy dependent systems. Studies involving several types of cell cultures have shown that NAA is present mainly in neurons. A reduction in the concentration of this compound has been interpreted as an indication of neuronal loss or dysfunction. ${ }^{12-14}$ In addition, NAA appears to be useful in assessing the extent of thalamic damage in patients in the vegetative state..$^{13}$

Abbreviations: Cho, choline; $\mathrm{Cr}$, creatine; $\mathrm{MRI}$, magnetic resonance imaging; MRS, magnetic resonance spectroscopy; NAA, N-acetyl aspartate 
Table 1 Clinical and demographic data and magnetic resonance findings in 14 patients with severe head injuries

\begin{tabular}{|c|c|c|c|c|c|c|c|c|}
\hline Patient & Age (years)/sex & Injury & GCS & $\begin{array}{l}\text { Examination lag after } \\
\text { injury (months) }\end{array}$ & Magnetic resonance findings & NAA: $\mathrm{Cr}$ & Cho: $\mathrm{Cr}$ & GOS \\
\hline 1 & $65 / M$ & MVA & 7 & 7 & $\mathrm{ICH}, \mathrm{DAl}$ & 1.76 & 1.25 & SD \\
\hline 2 & $40 / M$ & MVA & 4 & 6 & EDH, atrophy (right hippocampus) & 1.64 & 1.02 & $M D$ \\
\hline 3 & $18 / \mathrm{M}$ & MVA & 6 & 6 & $\mathrm{DAl}$ & 1.59 & 1.34 & $M D$ \\
\hline 4 & $20 / M$ & MVA & 6 & 7 & Multiple contusions, DAl, atrophy & 2.23 & 1.54 & SD \\
\hline 5 & $26 / F$ & Blunt trauma & 6 & 6 & DAl & 1.80 & 1.32 & SD \\
\hline 6 & $37 / M$ & Blunt trauma & 6 & 6 & $\mathrm{DAl}$ & 1.81 & 1.54 & SD \\
\hline 7 & $28 / M$ & MVA & 4 & 6 & DAl, skull fracture (depressed) & 1.36 & 1.16 & PVS \\
\hline 8 & $42 / F$ & MVA & 4 & 7 & $\mathrm{ICH}$ & 1.36 & 1.24 & PVS \\
\hline 9 & $32 / M$ & MVA & 6 & 6 & Multiple contusions, DAl, atrophy & 1.55 & 1.3 & PVS \\
\hline 10 & $56 / \mathrm{F}$ & Blunt trauma & 5 & 6 & Multiple contusions, DAl, atrophy & 0.75 & 1.79 & PVS \\
\hline 11 & $56 / \mathrm{F}$ & Blunt trauma & 5 & 8 & DAl & 0.95 & 0.96 & PVS \\
\hline 12 & $40 / M$ & MVA & 5 & 6 & Multiple contusions, DAl, atrophy & 1.17 & 1.42 & PVS \\
\hline 13 & $12 / M$ & MVA & 5 & 7 & DAl & 1.07 & 1.9 & PVS \\
\hline 14 & $35 / F$ & MVA & 5 & 6 & Multiple contusions, DAI & 1.17 & 1.53 & PVS \\
\hline
\end{tabular}

Cho, choline; Cr, creatine; DAl, diffuse axonal injury; EDH, extradural haematoma; F, female; GCS, Glasgow coma scale; GOS, Glasgow outcome scale; $I C H$, intracerebral haematoma; $M$, male; $M D$, mild disability; MVA, motor vehicle accident; NAA, N-acetyl aspartate; PVS, persistent vegetative state; SD, severe disability.

We present our initial results obtained from MRS thalamic examinations of six patients who regained awareness after being in the vegetative state and of eight patients in a persistent vegetative state.

\section{MATERIALS AND METHODS \\ Patient population}

Fourteen patients determined to be in vegetative state following severe traumatic brain injury were selected from the intensive care unit of the University of Istanbul's Cerrahpasa Medical School for retrospective evaluation. The group consisted of nine male and five female patients aged 12 to 65 years (mean 36 years). Patient admission records reported the nature of the trauma and the patient's age, sex, Glasgow coma scale score, and neurological status. We analysed the data available from the intensive care unit's clinical charts and all available follow up records. The senior author evaluated each patient. For each patient, we recorded the duration of the post-traumatic period and noted changes in neurological status according to the Glasgow outcome scale. For patients who regained awareness, we also determined the length of the recovery period.

Vegetative state was diagnosed according to the following criteria: (a) no evidence of awareness of self or environment, and no ability to interact with others; (b) no evidence of sustained, reproducible, purposeful, or voluntary behavioural responses to visual, auditory, tactile, or noxious stimuli; (c) no evidence of language comprehension or expression; (d) intermittent wakefulness manifested by the presence of sleepwake cycles; (e) sufficiently preserved hypothalamic and brainstem autonomic function to permit survival with medical and nursing care; (f) bowel and bladder incontinence; and $(\mathrm{g})$ variably preserved cranial nerve and spinal reflexes. ${ }^{5}$ Patients who remained in the vegetative state for at least six months after trauma were considered to be in a persistent vegetative state.

According to their state of consciousness during the study, we divided the patients into two groups. Group A consisted of all patients in a persistent vegetative state $(\mathrm{n}=8)$, and group $\mathrm{B}$ comprised those who had regained awareness after being in the vegetative state $(n=6)$. All MRS studies were performed after the sixth month of the vegetative state in both group A and group B patients. Table 1 shows the lag between the head injury and MRS study for both groups of patients. Five healthy volunteers who were within the patients' age range were evaluated as a control group (group C). We checked magnetic resonance imaging (MRI) results of both group A and group B patients before the MRS studies. Those with haemorrhagic and non-haemorrhagic lesions were excluded from this study. Patients in both groups with a normal appearing thalamus were included in this study. Thalamic atrophy was not an exclusion criterion.

\section{MRI and MRS procedures}

Once all the data were collected from the records, we performed MRI and MRS examinations on each patient according to standardised protocols. With the exception of the control group, all those studied underwent MRI while under general anaesthesia and receiving respiratory support from a mechanical ventilator. MRI was performed using a 1.5 T Signa system (GE Medical Systems, Waukesha, Wisconsin, USA) with version 5.3 software, using a clinical protocol that includes spin echo $\mathrm{Tl}$ weighted images, gradient echo T2 images, and fast spin echo T2 weighted images from a minimum of two different orthogonal planes. The standard head coil was used for image guided single voxel spectroscopic acquisition. All single voxel proton spectroscopy studies were obtained with a $1.5 \mathrm{~T}$ clinical scanner using PROBE/SV software (GE Medical Systems) with automated shimming, water suppression, and data processing capabilities. Axial Tl weighted MRIs were used to prescribe rectangular voxels of the thalamus while minimising cerebrospinal fluid contamination (fig lA). Axial Tl weighted MRIs (fig lB) were used to determine that there were no abnormalities on the thalami. The voxel dimensions used in all examinations (in both group
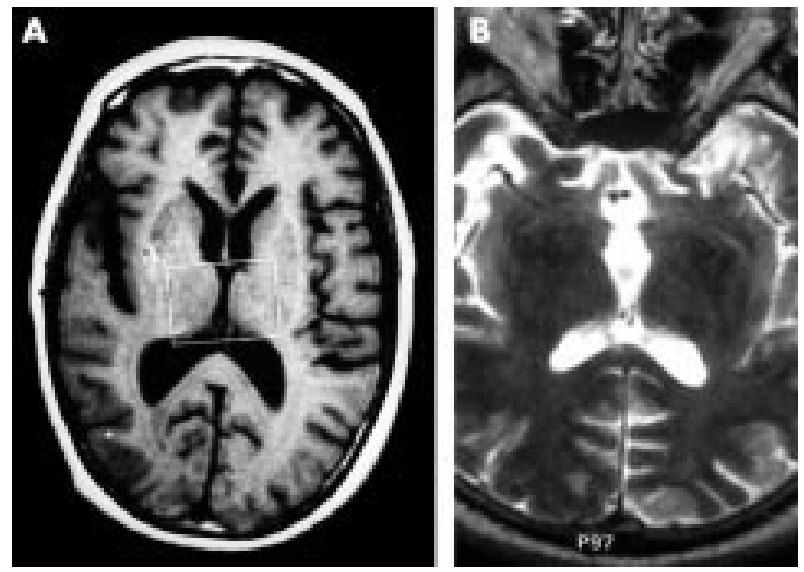

Figure 1 Axial T1 weighted magnetic resonance image shows (A) a typical site of magnetic resonance spectroscopic (MRS) acquisition of the thalamus (rectangle) and (B) no haemorrhagic or non-haemorrhagic lesions in both thalami. 

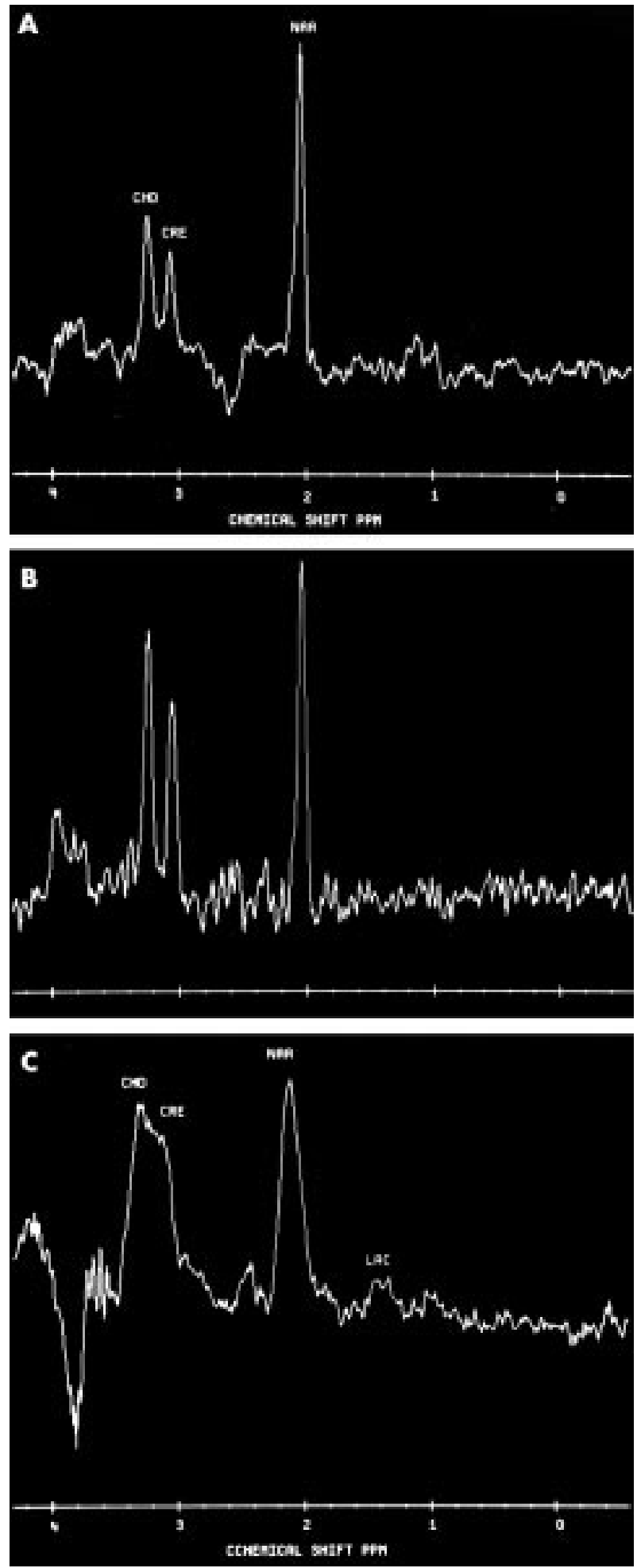

Figure 2 MRS spectra obtained (A) from the bilateral thalamus of a healthy volunteer, (B) a patient who regained awareness by the time the study was concluded, and (C) a patient in a persistent vegetative state.

A and group B) routinely prescribed from the axial image approximated A (anterior/posterior) $\times \mathrm{B}$ (right/left) $\times \mathrm{C} \mathrm{mm}$ (superior/inferior). The voxel locations were carefully chosen by one of the investigators ( $\mathrm{S} \mathrm{A}$ ) to ensure the same location in each patient. However, there is some cerebrospinal fluid in all patient voxel volume and this cerebrospinal fluid contamination is inevitable in our voxel volume and our technique

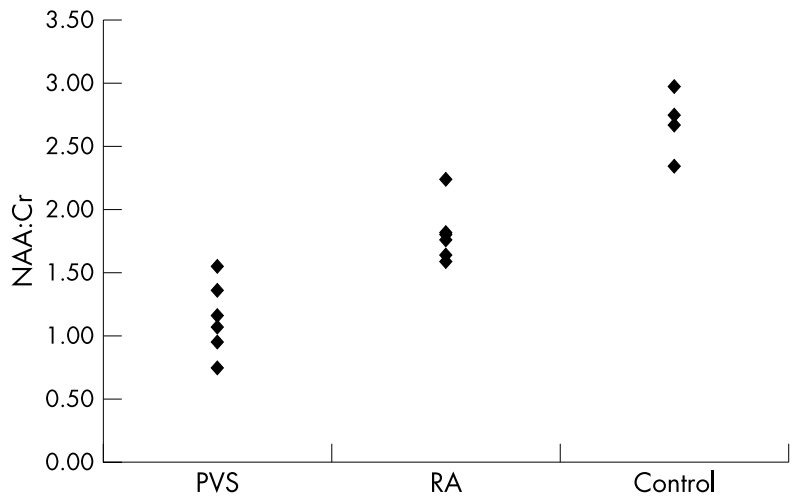

Figure 3 Dataplot comparing $\mathrm{N}$-acetyl aspartate to creatine (NAA:Cr) ratios of the thalamus in patients in a persistent vegetative state (PVS; $1.17(0.25))$, patients who regained awareness after being in the vegetative state (RA; $1.80(0.26))$ and the control group (2.67 (0.26)). PVS $v$ RA: $p<0.001$; PVS $v$ control: $p<0.001$; RA $v$ control: $p<0.001$.

(single voxel technique). Careful shimming is necessary to minimise line widths of spectra from this region. Water suppression was achieved by using three chemical shiftselective radiofrequency pulses followed by a dephasing gradient applied to each of three axes. All patients' long echo time spectra were obtained using point resolved spectroscopy (repetition time $1500 \mathrm{~ms}$, echo time $270 \mathrm{~ms}, 128$ acquisitions). Gradient shimming on the voxel and optimisation of the solvent suppression were performed before the start of acquisition. The spectral acquisition time per voxel was approximately six minutes. The spectral processing was performed using commercially available software (ProNMR, Softpulse Software, Guelph, Ontario, Canada) using zero filling to $8 \mathrm{~K}$ data points, $2 \mathrm{~Hz}$ line broadening applied in the time domain, one dimensional Fourier transformation, and zero order phase correction. Areas under the peaks were estimated using a Marquardt fitting routine to Lorentzian line shapes in the frequency domain. Using this method, we calculated peak ratios for the metabolites. Ratios of metabolites relative to $\mathrm{Cr}$ were calculated and compared with the values obtained in controls within the same age range (fig 2).

\section{Statistical analysis}

The metabolite ratios in the control and patient groups were compared by analysis of variance. Probability values $<0.05$ were considered significant and $\mathrm{p}<0.001$ was considered highly significant. Data are mean (SD).

\section{RESULTS}

\section{Patient characteristics}

The majority of the patients were relatively young. The mean ages in group A, group B, and group C were statistically similar, at 38.0 (15.7) years, 33.8 (19.0) years, and 37.0 (18.0) years, respectively $(\mathrm{p}=0.68)$. Patients in group A had lower Glasgow coma scale scores on admission. Among patients who regained awareness, the lengths of the recovery period were $117,81,78,96,141$, and 126 days (mean (SD) 106.5 (25.5) days) after the beginning of the vegetative state. Table 1 lists patient demographic data.

\section{MRI and MRS findings}

In the majority of the patients evaluated $(85.7 \%$ of group A and $80.0 \%$ of group B), MRI examinations of the brain showed haemorrhagic or non-haemorrhagic diffuse axonal injury. The lesions detected varied in size and were most often found in the corpus callosum, cerebral white matter, brainstem, and cerebellar hemispheres. MRI examinations also showed a normal thalamus in patients in groups A, B, and C. Generalised 


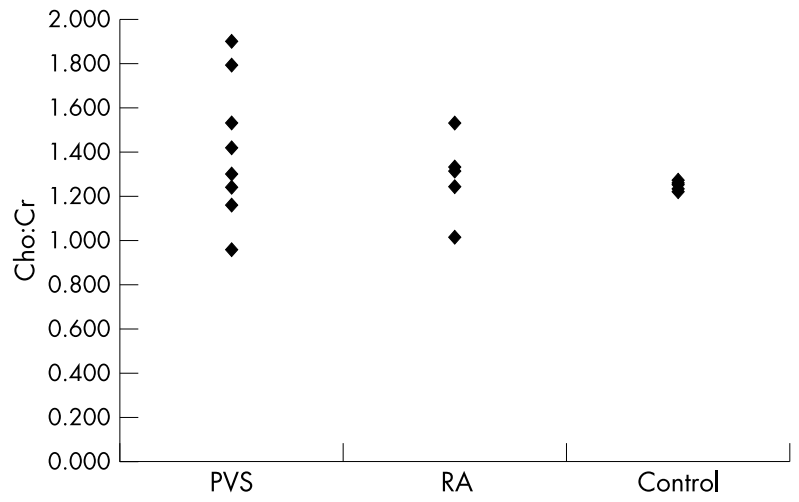

Figure 4 Dataplot comparing choline to creatine (Cho: $\mathrm{Cr}$ ) ratios of the thalamus in PVS $(1.41(0.31))$, RA (1.33 (0.19)), and control groups (1.26 (0.02)).

cerebral atrophy was noted on MRI (obtained late in the posttrauma phase) of all patients, regardless of the patient's neurological status at the time of the examination.

MRS showed an NAA:Cr ratio of $1.17(0.25)$ in patients in group $\mathrm{A}, 1.80(0.26)$ in patients in group $\mathrm{B}$, and $2.67(0.26)$ in the control group (fig 3 ). The ratios were significantly lower in both group A and group B than in group $\mathrm{C}(\mathrm{p}<0.001)$. However, the NAA:Cr of group A was found to be significantly lower than that of group B $(p<0.001)$. Comparison of the Cho:Cr ratios of all groups, however, showed no significant differences (fig 4). MRI showed lesions of the brainstem in four group A patients (50\%) and in four patients who eventually regained consciousness (67\%). There was no correlation between the presence of a brainstem lesion and a patient's state of awareness $(p=0.7)$.

\section{DISCUSSION}

Over the past 30 years, medical and technical advances have led to a steady decline in the mortality associated with severe traumatic brain injury, with an impressive reduction of approximately $10 \%$ per decade. ${ }^{16}$ Although these statistics are promising, the fact remains that up to $14 \%$ of severe traumatic brain injury victims remain in the vegetative state after their injury, ${ }^{17}$ a condition among the most devastating and morally challenging in modern medicine. ${ }^{18}{ }^{19}$ Potential outcomes of the vegetative state range from complete recovery to death, with virtually no clinical or instrumental method of accurately predicting where a particular patient will fall within this range. Obviously, there is a need for some sort of clinical or instrumental advance to predict the potential outcome for patients in the vegetative state-not only would such an advance facilitate the treatment of these patients, it would also provide physicians and families with additional pertinent information that would potentially ease the moral and emotional dilemma of caring for the patients in the vegetative state.

Correctly diagnosing the vegetative state is the first and one of the most crucial steps in forming a preliminary prediction of a patient's potential for recovery and it is essential in making well informed decisions regarding patient care. Although many studies have examined the anatomical and physiological bases of consciousness and awareness, ${ }^{26}$ current research has not provided us with a good understanding of the nature and location of brain lesions that cause a vegetative or persistent vegetative state. Awareness refers to the collective thoughts and feelings of the patient and denotes the knowledge of one's own existence, sensations, and cognition in the external and internal worlds. Vegetative state refers to a state of wakefulness without demonstrable awareness and thus raises basic questions about the nature of brain pathology, which can lead to a dissociation of awareness from arousal. ${ }^{6}$ This dissociation suggests that the two components of consciousness (awareness and arousal) are mediated by separate and distinct anatomical structures. Cerebral structures that are essential for human awareness are the cortex, white matter, and thalamus. Almost all information from the external world reaches the cerebral cortex by way of the thalamic nuclei, which also receive reciprocal projections from the cerebral cortex. ${ }^{6}$ All sensory stimuli (with the exception of olfactory stimuli) are projected through specific thalamic nuclei to the sensory cortex, with visual and somatosensory stimuli projected as point to point receptive fields. ${ }^{6}$

Postmortem examinations in a large series of patients in a post-traumatic persistent vegetative state ${ }^{20} 21$ find varying degrees of destruction, including degeneration that affects the cerebral cortex bilaterally, the cerebral white matter, and occasionally the mesencephalic tegmental structures. These structures may be affected independently or simultaneously. The mesencephalic lesions are primarily the result of damage secondary to early compression of the brainstem that results from swelling due to brain injury (herniation). In addition to cases of widespread damage due to anoxic or traumatic brain injury, vegetative states may also result from focal injuries to the paramedian brainstem and thalamus. ${ }^{22-25}$ Postmortem studies of non-traumatically induced persistent vegetative state are documented less frequently within the literature; however, these studies also report findings of multifocal bilateral cerebral lesions with and without severe destruction of the basal ganglia or thalamus. ${ }^{26}$

The investigation of Jennett et $a l^{27}$ on the neuropathology of vegetative and severely disabled patients after head injury reported that diffuse axonal injury was less common in severely disabled patients than in patients in the vegetative state $(50 \% \vee 80 \%)$, with the difference most pronounced in cases of the most severe lesions ( $17 \%$ v 63\%). Likewise, structural abnormalities in the thalamus were much less common among severely disabled patients than among patients in the vegetative state $(37 \% v 80 \%)$. In addition, thalamic abnormality increased in patients who remained in the vegetative state for longer than three months (37\% v 96\%), by which time transneuronal thalamic degeneration subsequent to diffuse axonal injury could be identified microscopically. Transneuronal degeneration suggests that an injury sustained at one nervous system locale can affect distant sites that are interconnected with the damaged area. ${ }^{28-31}$ For example, if a lesion within the cortical tissue interrupts the neuronal connections between the cerebral cortex and the thalamus, thalamic atrophy may result and target cells in the thalamus may die due to decreased trophic support. The neuropathology studies of Jennett et $a l^{27}$ indicate that such a relation exists between cortical and thalamic integrity in patients in a persistent vegetative state. Accordingly, it may be inferred that the integrity of subcortical structures may be compromised more significantly when cortical lesions are present. Not surprisingly, therefore, transneuronal degeneration or direct traumatic lesion is evident in almost all (96\%) patients in a persistent vegetative state at approximately three months after sustaining the initial brain trauma. ${ }^{27}{ }^{32}$ Since cortical diffuse axonal injury is transneuronal in origin and takes approximately three months to appear, detection of structural changes in the thalamus in association with cortical diffuse axonal injury depends on how long a patient survives while in the vegetative state..$^{27} 32$

A reliable assessment of prognosis in the vegetative state following cerebral anoxia is crucial in making decisions concerning initiation or prolongation of extended intensive care procedures. Clinical signs (such as duration of coma), laboratory findings (such as serum concentrations of neuron specific enolase), and functional tests permit only indirect estimation of the extent of structural brain damage. ${ }^{33}$ Current radiographic examinations, including computed tomography and conventional MRI, also cannot provide a definite prognosis during the early or late stages of the vegetative state. ${ }^{33}$ The 
microscopic grades of diffuse axonal injury and transneuronal thalamic degeneration that result from cortical diffuse axonal injury are undetectable by conventional neuroimaging techniques; however, it is possible that transneuronal thalamic degeneration is responsible for at least some of the clinically evident neurological and cognitive deficits of patients in a persistent vegetative state. Although neuropathology studies can detect transneuronal degeneration or direct traumatic lesions present three months after trauma in nearly all (96\%) patients in a persistent vegetative state, MRI studies conducted by Kampfl et $a l^{17}$ showed thalamic damage with haemorrhagic or non-haemorrhagic lesions in only $40 \%$ of patients in a persistent vegetative state. They did not evaluate thalamic atrophy in their study.

MRS, with its ability to detect in vivo the neurochemical alterations associated with various pathologies, has the potential to fill this role. MRS can measure the concentrations of neurochemicals such as NAA, Cr, and Cho and provide a kind of chemical illustration of the brain's composition. In grey matter, NAA is found in neuronal cell bodies, whereas in white matter it is found predominantly in axons. ${ }^{12} 133435 \mathrm{~A}$ lowered NAA peak is associated with various pathologies, suggesting that neuronal or axonal loss or dysfunction would be detectable by MRS in the form of reduced NAA concentrations. ${ }^{156}$ In models of brain injury in the rat, Rubin et $\mathrm{al}^{37}$ reported a loss of NAA in the cortex of animals one hour after injury and Signoretti et $a l^{38}$ reported a similar decrease within minutes of injury. In a swine model of rotational acceleration injury, Cecil et $a l^{39}$ also noted decreased NAA concentrations one hour after injury, with concentrations remaining stable up to one week after the injury.

Few researchers have used proton MRS for the detection of diffuse axonal injury in patients with head trauma, although the few investigations that do appear in the literature are promising in terms of the role of proton MRS. Choe et $a l^{36}$ evaluated neuronal and axonal dysfunction by using in vivo proton MRS in patients with closed head injury and Ricci et $a l^{15}$ found greatly decreased NAA:Cr and NAA:Cho ratios in the frontal cortices of a series of 14 patients in vegetative state, despite normal MRI results in this region. These studies suggest both that the observed reduced NAA:Cr ratio may be an indicator of axonal loss in patients with head injury and that lower NAA:Cr ratios correlate with poor clinical outcome.

Evaluating the usefulness of thalamic proton MRS for patients in vegetative state was the primary goal of this study. Because thalamic abnormality results from both primary damage to the structure itself and secondary effects (transneuronal degeneration from cortical lesion detectable three months after trauma) of the persistent vegetative state, ${ }^{27}{ }^{32} \mathrm{a}$ precise radiographic evaluation of this area is an excellent method for determining the extent of axonal and neuronal damage in patients in the vegetative state. The MRS results that we obtained showed that both patients in a persistent vegetative state and those who regained awareness by the time the study was concluded had significantly lower NAA:Cr ratios than the control group. When the two brain injured groups were compared, those in a persistent vegetative state had lower NAA:Cr ratios than the group that eventually regained awareness. On the basis of this comparison, NAA:Cr ratios appear to be relevant to the differentiation between patients in vegetative state who have the potential for some degree of recovery and those for whom any recovery is unlikely. Our results showed that low NAA:Cr ratios are indicative of neuronal and axonal loss and secondary transneuronal degeneration in all patients in a persistent vegetative state at the sixth month; however, these same changes were evident in neuropathological studies as early as three months after the initial trauma in almost all (96\%) patients who had been in vegetative state. Our results indicate that metabolite ratios routinely measured with MRS furnish a means to quantify brain metabolic damage, allowing for the possibility of grading the severity of neuronal and axonal injury in patients in the vegetative state.

Our study has some technical limitations. Firstly, we used a large voxel size for MRS examination of the thalamus and this may cause some degree of cerebrospinal fluid contamination. Cerebrospinal fluid contamination may have been higher in the persistent vegetative state group than in the vegetative state group and this situation may cause some degree of miscalculation of brain metabolites with MRS. Secondly, we used the single voxel technique in this study. However, single voxel localisation techniques have a number of limitations in the context of clinical spectroscopy: voxel sizes are often relatively large and no information is available concerning spatial distribution or extent of metabolic abnormalities. In contrast, MRS imaging has significant advantages in terms of spatial resolution, efficiency of data collection, and mapping the distribution of cerebral metabolite concentrations. We are planning to use the MRS imaging technique and a small voxel size in our future thalamic magnetic spectroscopy study in patients in a persistent vegetative state.

Although we do feel that our initial results with thalamic proton MRS imaging are promising and that thalamic proton MRS may provide the possibility of grading the severity of neuronal and axonal injury in the vegetative state, we recognise that certain aspects of our study can be perceived as limitations. MRS imaging was not performed in the early vegetative state stage in all patients; rather, we waited at least three months in order to be able to detect transneuronal degeneration and secondary thalamic involvement. A more serious limitation to our study was the small size of our patient population. Without a larger number of patients, we cannot make any definitive statements about the value of thalamic proton MRS for the evaluation of patients in vegetative state. Studies of larger and more homogeneous (in terms of duration of the vegetative state) patient populations and using new MRS techniques (such as MRI) would improve our ability to define and evaluate the future role of thalamic proton MRS in forming a prognosis and predicting an eventual outcome.

\section{ACKNOWLEDGEMENTS}

The authors thank Civan Islak MD for his efforts in preparing this manuscript.

\section{Authors' affiliations}

M Uzan, S G R Dashti, S Aydin, M Hanci, C Kuday, Department of Neurosurgery, Cerrahpasa Medical School, Istanbul University, Istanbul, Turkey

S Albayram, Department of Radiology, Cerrahpasa Medical School, Istanbul University, Istanbul, Turkey

Competing interests: none declared

\section{REFERENCES}

1 Jennett B, Plum F. Persistent vegetative state after brain damage. Lancet $1972 ; \mathrm{i}: 734-7$

2 Kampfl A, Schmutzhard E, Franz G, et al. Prediction of recovery from post-traumatic vegetative state with cerebral magnetic-resonance imaging. Lancet 1998;351:1763-7.

3 Uzan M, Bozkus $H$, Hanci $M$, et al. The prognosis of vegetative state after head injury. Turk Neurosurg 1997;7:60-4.

4 Celesia GG. Persistent vegetative state. Neurology 1993;43:1457-8.

5 Quality Standards Subcommittee of the American Academy of Neurology. Practice parameters: assessment and management of patients in the persistent vegetative state. Neurology 1995;45:1015-8. 6 Kinney HC, Samuels MA. Neuropathology of the persistent vegetative state. A review. J Neuropathol Exp Neurol 1994;53:548-58.

7 Castillo M, Kwock L. Proton MR spectroscopy of common brain tumors. Neuroimaging Clin N Am 1998;8:733-52.

8 Cecil KM, Lenkinski RE. Proton MR spectroscopy in inflammatory and infectious brain disorders. Neuroimaging Clin N Am 1998;8:863-80.

9 Demaerel P, Van Hecke P, Van Oostende S, et al. Bacterial metabolism shown by magnetic resonance spectroscopy. Lancet 1994;344:123443 
10 Gadian DG, Shaw D, Moonen CTW, et al. Advances in proton magnetic resonance spectroscopy of the brain. A report on a workshop held in Oxford, England December 16-18, 1992. Magn Reson Med 1993;30: 1-3.

11 Wang ZJ, Zimmerman RA. Proton MR spectroscopy of pediatric brain metabolic disorders. Neuroimaging Clin N Am 1998;8:781-807.

12 Castillo M, Kwock L, Muckhreji SK. Clinical applications of proton MR Spectroscopy. Am J Neuroradiol 1996;17:1-15.

13 Cecil KM, Hills EC, Sandell ME, et al. Proton magnetic resonance spectroscopy for the detection of axonal injury in the splenium of the corpus callosum of brain-injured patients. J Neurosurg 1998:88:795-801.

14 Michaelis T, Merboldt KD, Bruhn H, et al. Absolute concentrations of metabolites in the adult human brain in vivo: quantification of localized proton MR spectra. Radiology 1993;187:219-27.

15 Ricci R, Barbarella G, Musi P, et al. Localised proton MR spectroscopy of brain metabolism changes in vegetative patients. Neuroradiology 1997;39:313-9.

16 Bullock MR, Chesnut RM, Clifton G, et al. Guidelines for the management of severe head injury. Eur J Emerg Med 1996:3:109-27.

17 Kampfl A, Franz G, Aichner F, et al. The persistent vegetative state after closed head injury: clinical and magnetic resonance imaging findings in 42 patients. J Neurosurg 1998;88:809-16.

18 Payne K, Taylor RM, Stocking C, et al. Physicians' attitudes about the care of patients in the persistent vegetative state: a national survey. Ann Intern Med 1996;125:104-10.

19 Zeman A. Persistent vegetative state. Lancet 1997;350:795-9.

20 Danze F, Brule JF, Haddad K. Chronic vegetative state after severe head injury: clinical study; electrophysiological investigations and CT scan in 15 cases. Neurosurg Rev 1989;12(suppl 1):477-99.

21 Adams JH, Jennett B, McLellan DR, et al. The neuropathology of the vegetative state after head injury. J Clin Pathol 1999;52:804-6.

22 Relkin NR, Petito CK, Plum F. Coma and the vegetative state associated with thalamic injury after cardiac arrest. Ann Neurol 1990;28:221-6.

23 Facon $M$, Steriade $M$, Wertheim $N$. Hypersomie prolonge engendere par des lesions bilatereles du systeme activateur medial. Le syndrome thrombotique de la bifurcation du tronc basilaire. Rev Neurol 1958;98:117-33.

24 Castaigne $\mathbf{P}$, Lhermitte $F$, Buge $A$, et al. Paramedian thalamic and midbrain infarct: clinical and neuropathological study. Ann Neurol $1981 ; 10: 127-48$
25 Kinney HC, Korein J, Panigrahy A, et al. Neuropathological findings in the brain of Karen Ann Quinlan. The role of the thalamus in the persistent vegetative state. N Engl J Med 1994;330: 1469-75.

26 Dougherty JH Jr, Rawlinson DG, Levy DE, et al. Hypoxic-ischemic brain injury and the vegetative state: clinical and neuropathologic correlation. Neurology 1981;31:991-7

27 Jennett B, Adams JH, Murray LS, et al. Neuropathology in vegetative and severely disabled patients after head injury Neurology 2001;56:486-90.

28 Jessell TM. Reactions of neurons to injury. In: Kandel ER, Schwartz HJ, lessell TM, eds. Principles of neuronal science, 3rd ed. New York: Elsevier Science Publishing, 1991:258-69.

29 Matthews MA. Death of the central neuron: an electron microscopic study of thalamic retrograde degeneration following cortical ablation. $J$ Neurocytol 1973;2:265-88.

30 Ross DT, Ebner FF. Thalamic retrograde degeneration following cortical injury: an excitotoxic process? Neuroscience 1990;35:525-50.

31 Ross DT, Graham DI, Adams JH. Selective loss of neurons from the thalamic reticular nucleus following severe human head injury. J Neurotrauma 1993;10:151-65.

32 Adams JH, Graham DI, Jennett B. The neuropathology of the vegetative state after an acute brain insult. Brain 2000;123:1327-38.

33 The Multi-Society Task Force on PVS. Medical aspects of the persistent vegetative state (1). N Engl J Med 1994;330:1499-508

34 Jacobson KB. Studies on the role of $\mathrm{N}$-acetylaspartic acid in mammalian brain. J Gen Physiol 1959:43:323-3.

35 Tallan $\mathbf{H H}$. Studies on the distribution of $\mathrm{N}$-acetyl-L-aspartic acid in brain. J Biol Chem 1956;224:41-5

36 Choe BY, Suh TS, Choi KH, et al. Neuronal dysfunction in patients with closed head injury evaluated by in vivo $1 \mathrm{H}$ magnetic resonance spectroscopy. Invest Radiol 1995;30:502-6.

37 Rubin Y, Cecil K, Wehrli S, et al. High-resolution IH NMR spectroscopy following experimental brain trauma. J Neurotrauma 1997; 14:441-9.

38 Signoretti S, Marmarou A, Tavazzi B, et al. N-acetylaspartate reduction as a measure of injury severity and mitochondrial dysfunction following diffuse traumatic brain injury. J Neurotrauma 2001;18:977-91.

39 Cecil KM, Lenkinski RE, Meaney DF, et al. High-field proton magnetic resonance spectroscopy of a swine model for axonal injury. J Neurochem 1998:70:2038-44.

CiteTrack will alert you by email whenever new content in the Journal of Neurology, Neurosurgery, and Psychiatry or a participating journal is published that matches criteria you want to track Topics: Tell CiteTrack which words or subjects to watch for in new content Authors: Be alerted whenever key authors you are following publish a new paper Articles: Know whenever a paper of interest to you is referenced by another paper

\section{www.jnnp.com}

